\title{
Comportamientos de riesgo para la salud en hombres mayores brasileños viudos
}

\author{
Health risk behaviors in elderly Brazilian widowers
}

${ }^{1}$ Magíster en Salud Colectiva. Programa de Saúde Coletiva, Universidade Estadual de Londrina, Londrina, Brasil. $\triangle$ id

${ }^{2}$ Doctor en Salud Colectiva. Programa de Pós Graduação em Saúde Coletiva, Universidade Estadual de Londrina, Londrina, Brasil. $\bowtie$ iD
RESUMEN Se buscó comparar la prevalencia de comportamientos de riesgo para la salud en hombres mayores viudos con las de hombres mayores con pareja, solteros y divorciados/separados, así como la prevalencia de comportamientos de riesgo en hombres mayores viudos según rango de edad, escolaridad y raza/color. Se realizó un estudio transversal con hombres mayores ( $\geq 60$ años) entrevistados por el Sistema de Vigilancia de Factores de Riesgo y Protección para Enfermedades Crónicas por Encuesta Telefónica (Vigitel) en $2016(n=5.384)$ y $2017(n=5.801)$ que incluye muestras representativas de adultos de las capitales de los estados brasileños y del Distrito Federal. De ellos, 886 eran viudos. Los comportamientos de riesgo fueron: inactividad física en el tiempo libre, consumo irregular de frutas, verduras y legumbres, tabaquismo y consumo abusivo de alcohol. La prevalencia de tabaquismo fue menor entre los hombres mayores con pareja $[R P=0,68$; IC95\% $(0,52$ $0,90)$ ] que entre los viudos. En los demás comportamientos de riesgo no se observaron diferencias en la prevalencia de los viudos en relación a los demás grupos. Cuando se analizaron solamente los viudos, se observaron importantes asociaciones de los comportamientos de riesgo con las variables grupo de edad y escolaridad, pero no con raza/color. PALABRAS CLAVES Salud de la Persona Mayor; Estilo de Vida; Estudios Transversales; Viudez; Envejecimiento; Brasil.

ABSTRACT The objective of this study was to compare the prevalence of health risk behaviors among elderly widowers with that of elderly partnered males, as well as single or divorced/separated men. Additionally, we set out to examine the prevalence of risk behaviors in elderly widowered men according to age, education level, and race/skin color. A cross-sectional study was conducted with elderly men ( $\geq 60$ years) who were interviewed through the Surveillance System of Risk and Protective Factors for Chronic Diseases by Telephone Survey (Vigitel) in $2016(n=5,384)$ and $2017(n=5,801)$. The Vigitel survey includes representative samples of adults residing in the capitals of Brazilian states and the Federal District, and of those surveyed, 886 were widowers. Identified risk behaviors included physical inactivity during leisure time, irregular consumption of fruits, vegetables and legumes, smoking and alcohol abuse. The prevalence of smoking was lower among partnered elderly men $[\mathrm{PR}=0.68, \mathrm{Cl} 95 \%(0.52-0.90)]$ than among widowers. There were no differences in the prevalence of other risk behaviors between widowers and other groups. When only widowers were taken into account, there was significant association of risk behaviors with age and educational level, but not with race/skin color.

KEY WORDS Health of the Elderly; Life Style; Cross-Sectional Studies; Widowhood; Aging; Brazil. 


\section{INTRODUCCIÓN}

Las transiciones demográficas y epidemiológicas observadas en Brasil en las últimas décadas han influido en el envejecimiento de la población, especialmente, en el aumento del número y proporción de los adultos mayores. Asociado a estas transiciones, otro cambio importante en el perfil de la población brasileña está relacionado con la situación conyugal. Entre 2000 y 2010 hubo un aumento en la proporción de viudos, divorciados/separados y solteros. En 2010, Brasil tenía alrededor de ocho millones de viudos, lo que representa el $4 \%$ de la población, es decir, a medida que la población envejece, el número de viudos aumenta ${ }^{(1,2)}$. Además, otro cambio se ha relacionado con el perfil de morbilidad y mortalidad en el país, con el protagonismo de las enfermedades crónicas no transmisibles $(E C N T)^{(3,4,5)}$. La mayoría de estas enfermedades se relacionan con comportamientos de salud, como la inactividad física en el tiempo libre; el consumo irregular de frutas, verduras y legumbres; el tabaquismo; y el abuso del alcohol.

En general, los estudios indican que los hombres se involucran más en comportamientos de riesgos y acceden menos a los servicios de salud, factores asociados al incremento de condiciones graves y crónicas y a mayores índices de mortalidad que las mujeres $^{(6,7,8)}$. Por lo tanto, también es importante considerar la dimensión del género masculino, y las singularidades y diversidades en las relaciones sociales más amplias que se vinculan con estas diferencias como, por ejemplo, la construcción en torno a lo masculino que se relaciona con ser más fuerte, más varonil y solo buscar servicios de salud en condiciones más severas. Además, según Gomes et al. ${ }^{(9)}$ los servicios de salud, especialmente en atención primaria, están más dirigidos a la salud de las mujeres.

Buscando abordar esta situación, el Ministerio de Salud de Brasil lanzó, en 2009, la Política Nacional de Atención Integral a la Salud de los Hombres (PNAISH) con los objetivos de promover la mejora de las condiciones de salud de la población masculina de
Brasil y contribuir a la reducción de la morbilidad y la mortalidad, intentando facilitar el acceso a los servicios de salud ${ }^{(10)}$.

Uno de los factores que parece estar relacionado con la salud de los hombres es la situación conyugal. Según algunos estudios, las personas casadas o en una unión estable tienen mejores indicadores de salud en comparación con divorciados/separados, solteros y viudos ${ }^{(11,12,13)}$. Estos resultados se explican, al menos en parte, por el factor protector relacionado con una red de apoyo social más grande, apoyo económico y psicológico. Otro factor es la selección, por la cual los sujetos con mejor salud y condiciones económicas tienen más probabilidades de permanecer o tener una relación conyugal ${ }^{(7,11,12,13)}$. Además, estos factores positivos parecen ser más favorables para los hombres, en parte debido a la construcción social de los roles de género en la relación, en la que la compañera termina siendo responsable de realizar diversas funciones atribuidas a la atención con la sa$\operatorname{lud}^{(7,13,14,15)}$. Para Gomes ${ }^{(16)}$, la dependencia de los hombres al cuidado realizado por las mujeres, se refuerza de generación en generación y en la convivencia con el sexo opuesto.

Dado este contexto, la viudez también es una situación que parece influir en las condiciones de salud de las personas. Además de ser un evento recurrente en cualquier sociedad y estar presente en la vida de la mayoría de la población, su significado varía de acuerdo con los contextos sociales, culturales, económicos y personales, como el tiempo vivido con la pareja, la calidad de esta relación, edad y género ${ }^{(17,18)}$. La viudez es una condición socialmente construida para la mujer, porque la mayoría de las mujeres se casan con parejas mayores, tienen una esperanza de vida más larga que los hombres y buscan menos una nueva relación conyugal $^{(2,7,17)}$. Para Motta ${ }^{(19)}$, la viudez en los hombres es de baja incidencia, incluso entre los mayores y, además, a diferencia de las mujeres, la repercusión social es más tranquila, lo que favorece una mayor búsqueda de una nueva relación conyugal.

Por lo tanto, los efectos de la viudez en las condiciones de salud entre hombres $y$ 
mujeres parecen ser diferentes ${ }^{(20,21)}$. La evidencia indica que la viudez masculina se asocia con una mayor mortalidad ${ }^{(11,20,21)}$, una mayor adopción de comportamientos de riesgo relacionados con la salud $(22,23,24,25,26)$, una mayor prevalencia de hipertensión y diabetes $^{(27)}$ y mayores gastos relacionados con la salud ${ }^{(28)}$. A lo anterior se suma que la mayoría de los estudios epidemiológicos que investigan este tema se han realizado en $\operatorname{EEUU}^{(21,22,25)}$ o en Europa ${ }^{(23,26,28)}$. Al parecer, no existen estudios realizados en el contexto latinoamericano. A pesar de la importancia del tema, dado que la viudez es una condición recurrente en cualquier sociedad, parece haber pocos estudios epidemiológicos que aborden este tema, especialmente cuando se relacionan con la salud de los hombres pues, como se mencionó anteriormente, la viudez es una condición más frecuente en las mujeres. Otro factor importante está relacionado con el envejecimiento de la población observado en Brasil en los últimos años y la necesidad de comprender mejor los factores relacionados con la salud de las personas mayores, especialmente de los hombres, ya que tienen una expectativa de vida menor con relación a la mujer. Por lo tanto, estudios con este enfoque pueden permitir una visión más amplia del proceso de salud-enfermedad de los hombres mayores incluso para ayudar a planificar acciones de salud.

De este modo, los objetivos de este estudio fueron comparar la prevalencia de los comportamientos de riesgo para la salud de hombres mayores viudos, con la de hombres mayores con pareja, con solteros y con divorciados/separados, y también comparar la prevalencia de los comportamientos de riesgo en hombres mayores viudos conforme la edad, escolaridad y raza/color.

\section{METODOLOGIA}

Se realizó un estudio transversal basado en la población de hombres mayores entrevistados por el sistema de Vigilancia de factores de riesgo y protección para enfermedades crónicas por encuesta telefónica (Vigitel) en $2016(n=5,384)$ y $2017(n=5,801)$. Vigitel se implementó en 2006 y desde entonces se Ileva a cabo anualmente. Es una encuesta realizada, a través de telefonía fija, a personas de 18 años o más que viven en las capitales de los 26 estados brasileños y en el Distrito Federal. El proceso de muestreo de Vigitel es probabilístico y se realiza a través de los siguientes pasos: en primer lugar, se seleccionan por sorteo 5.000 líneas telefónicas residenciales en cada una de las 26 capitales estatales y el Distrito Federal y, luego, por cada línea se selecciona un adulto residente para responder el cuestionario. En el año 2016, se entrevistaron 53.210 sujetos y, en 2017, 53.034. Para garantizar la representatividad de la muestra de los adultos de las capitales, se utilizaron pesos postestratificación en las variables; género, edad y educación, buscando igualar la distribución sociodemográfica de la muestra en relación con la distribución estimada para la población adulta de cada capital. Se pueden ver más detalles en los informes anuales de Vigitel[ ${ }^{(29,30)}$.

Los cuestionarios de Vigitel de 2016 y 2017 abarcaron preguntas respecto de diferentes temas relacionados con las características sociodemográficas, comportamientos de salud, peso y altura autoinformados, autoevaluación del propio estado de salud y condiciones de morbilidad. Se decidió incluir estos dos años, en lugar de uno solo, para aumentar el poder del análisis estadístico.

\section{Variables}

\section{Inactividad física en el tiempo libre}

Se midió a través de preguntas sobre la práctica de ejercicio físico o deporte en los últimos tres meses. También se hicieron preguntas sobre la duración y la frecuencia semanal de esta práctica. De acuerdo con las recomendaciones de la Organización Mundial de la Salud $(\mathrm{OMS})^{(31)}$, aquellos que practicaban menos de 150 minutos por semana de actividad física de intensidad leve/moderada o menos de 75 minutos por semana de actividad vigorosa fueron considerados físicamente inactivos 
durante el tiempo libre. Para la construcción de este indicador se consideraron actividades de caminata de intensidad ligera/moderada, caminata en cinta, entrenamiento con pesas, aeróbicos acuáticos, gimnasia general, natación, artes marciales, ciclismo y voleibol; correr, correr en cinta, aeróbicos, fútbol, baloncesto y tenis se clasificaron como actividades de intensidad vigorosa.

\section{Consumo irregular de frutas y/o verduras y legumbres}

Se midió a través de dos preguntas sobre la frecuencia semanal de consumo de estos alimentos. Para frutas la pregunta fue "¿Cuántos días a la semana sueles comer frutas?" y, para verduras y legumbres, “¿Cuántos días a la semana sueles comer por lo menos un tipo de verdura o legumbre (lechuga, tomate, col, chayote, berenjena, zapallito italiano), sin contar la papa, yuca/mandioca o ñame?". Para ambas opciones la respuesta era: nunca; casi nunca; 1-2 días; 3-4 días; 5-6 días; todos los días. Se consideraron como consumo irregular aquellas que referían a un consumo menor a cinco días por semana, por lo menos en una de las dos preguntas $y$, para ser considerado consumo regular, debería haber respondido las opciones "5-6 días" o "todos los días" en ambas preguntas.

\section{Tabaquismo}

Se midió a través de una pregunta simple sobre si el sujeto hacía uso de tabaco. Se consideró fumador a aquel que respondió sí a la pregunta.

\section{Consumo abusivo de alcohol}

Se midió a través de una pregunta en la que se consultaba si el sujeto, en los últimos 30 días, había consumido, en un único día, más de cinco dosis de bebida alcohólica. Fue considerada como dosis una lata de cerveza, una copa de vino, una dosis de agua ardiente, de whisky o cualquier bebida destilada.

La variable independiente fue la situación conyugal. En el Vigitel los sujetos podrían responder las siguientes opciones de respuestas: viudo, casado, unión estable, divorciado/ separado o soltero. Para este estudio las categorías "casado" y "unión estable" fueron agrupadas como "con parejas".

Otras variables que fueron usadas en la estratificación de la muestra fueron: la escolaridad ( 0 a $8 ; 9$ a $11 ;$ y 12 años o más de estudio) el rango de edad (60 a 69; 70 a $79 ;$ y 80 años o más) y raza/color (blancos/amarillos y negros/pardos/indígenas).

\section{Análisis de datos}

La caracterización sociodemográfica de la muestra se realizó a través de los datos descriptivos y el análisis de frecuencia. Para calcular la prevalencia de comportamientos de riesgo relacionada con la salud, consideramos a todos los sujetos de la muestra, divididos en viudos, con pareja, divorciados/separados y solteros, estratificados por edad, educación y raza/color. Para este análisis usamos la prueba de chi-cuadrado y el valor de $p<0,05$.

Por último, se calculó la razón de prevalencia de los comportamientos de riesgo y se realizaron dos análisis. En el primero, se consideraron todas las situaciones conyugales, de acuerdo con el rango de edad, escolaridad y raza/color, siendo la viudez la categoría de referencia. En segundo lugar, se consideraron solo los viudos y la razón de prevalencia se calculó de acuerdo con el rango de edad (60 a 69 años como categoría de referencia), escolaridad ( $\geq 12$ años como categoría de referencia) y raza/color (blancos/amarillos como categoría de referencia). Además, para estos cálculos se utilizó el intervalo de confianza del $95 \%$.

El consentimiento libre e informado fue obtenido oralmente en el momento de la entrevista. El Vigitel fue aprobado por la Comisión Nacional de Ética en Investigación para Seres Humanos del Ministerio de Salud para cada año de estudio (CAAE 65610017.1.0000.0008). Si bien cualquier persona puede acceder por Internet al banco de datos de la investigación, no hay identificación de los entrevistados. 


\section{RESULTADOS}

Considerando la muestra completa, la mayoría eran sujetos con pareja (78,5\%). El 7,9\% de los hombres eran viudos. Se observó que el $74,8 \%$ de los viudos tenían 70 años o más, el 52,4\% tenía menos de nueve años de escolaridad y el $88,3 \%$ era de raza/color blanca o amarilla. Al comparar la caracterización de los viudos con las de los otros grupos, se observaron diferencias importantes en relación con la edad (en los otros grupos la mayoría tenía entre 60 y 69 años) y escolaridad (en los otros grupos, la mayoría tenía al menos 9 años de escolaridad) (Tabla 1).

Con relación a la inactividad física en el tiempo libre, no se observaron diferencias en las prevalencias de acuerdo con la situación conyugal en general, mientras que al ser estratificada por edad y escolaridad se observaron algunas diferencias. Entre los hombres de 80 años o más, la mayor prevalencia fue entre aquellos con pareja $(77,1 \%)$ y la menor fue entre divorciados/separados $(60,8 \%)$. Ya entre los hombres de menor escolaridad, la inactividad física en el tiempo libre fue mayor entre los que tenían pareja $(77,4 \%)$ y menor entre los solteros $(69,0 \%)$.

En general no fue observada asociación significativa entre el consumo irregular de frutas, verduras y legumbres con la situación conyugal. En la variable edad, sin embargo, se observó asociación en los hombres de 70 a 79 años: la mayor prevalencia fue entre los viudos $(62,4 \%)$ y la menor entre aquellos con pareja $(55,1 \%)$.

Ya en el caso del tabaquismo se observó una asociación significativa en el total de la muestra y también al ser estratificada por edad y escolaridad. En general, los divorciados/separados tuvieron la prevalencia más alta $(14,2 \%)$ y, aquellos con pareja, la más baja $(7,7 \%)$. Cuando se estratificó por grupo de edad, en las edades de 60 a 69 años, el tabaquismo también fue mayor entre los divorciados/separados $(17,9 \%)$ y menor entre aquellos con pareja $(9,5 \%)$. En los hombres de 70 a 79 años, el tabaquismo fue más frecuente entre los solteros $(11,6 \%)$ y más bajo en aquellos con pareja (5,7\%). En la variable escolaridad, quienes tenían de 9 a 11 años de escolaridad y 12 años o más, el tabaquismo fue mayor entre las personas solteras $(14,4 \%$ y

Tabla 1. Características sociodemográficas de hombres mayores ( $\geq 60$ años) residentes en capitales de los estados y Distrito Federal, general y estratificado por rango de edad y escolaridad, Vigitel, Brasil, 2016 y 2017 ( $n=11.185)$.

\begin{tabular}{|c|c|c|c|c|c|c|c|c|}
\hline \multirow[t]{2}{*}{ Variables } & \multicolumn{2}{|c|}{ Viudo } & \multicolumn{2}{|c|}{ Con pareja } & \multicolumn{2}{|c|}{$\begin{array}{c}\text { Divorciado/ } \\
\text { separado }\end{array}$} & \multicolumn{2}{|c|}{ Soltero } \\
\hline & $n$ & $\%$ & $\mathrm{n}$ & $\%$ & $\mathrm{n}$ & $\%$ & $n$ & $\%$ \\
\hline Total & 886 & 7,9 & 8.785 & 78,5 & 840 & 7,5 & 674 & 6,0 \\
\hline \multicolumn{9}{|l|}{ Rango de edad } \\
\hline 60 a 69 años & 224 & 25,3 & 4.990 & 56,8 & 509 & 60,6 & 449 & 66,6 \\
\hline 70 a 79 años & 346 & 39,1 & 2.991 & 33,1 & 257 & 30,6 & 181 & 26,9 \\
\hline 80 años 0 más & 316 & 35,6 & 884 & 10,1 & 74 & 8,8 & 44 & 6,5 \\
\hline \multicolumn{9}{|l|}{ Escolaridad } \\
\hline 0 a 8 años & 464 & 52,4 & 3.124 & 35,6 & 296 & 35,2 & 232 & 34,4 \\
\hline 9a 11 años & 193 & 21,8 & 2.378 & 27,1 & 240 & 28,6 & 208 & 30,9 \\
\hline 12 años o más & 229 & 25,8 & 3.283 & 37,4 & 304 & 36,2 & 234 & 34,7 \\
\hline \multicolumn{9}{|l|}{ Raza/color } \\
\hline Blanco/amarillo & 543 & 88,3 & 4.979 & 89,6 & 496 & 89,2 & 368 & 86,2 \\
\hline Negro/pardo/indígena & 72 & 11,7 & 581 & 10,4 & 60 & 10,8 & 59 & 13,8 \\
\hline
\end{tabular}


$16,7 \%$ respectivamente) y menor en aquellos con pareja $(7,1 \%$ y $6,3 \%$ respectivamente).

El consumo abusivo de alcohol fue asociado, tanto en general, como estratificado por escolaridad y raza/color. En general, la mayor prevalencia fue entre divorciados/separados $(15,1 \%)$ y la menor entre viudos $(8,2 \%)$; en los hombres con menor escolaridad (0 a 8 años) fue mayor entre solteros $(10,3 \%)$ y menor entre viudos $(5,4 \%)$; y con relación a la raza/color, en los blancos/amarillos fue mayor entre divorciados/separados $(14,1 \%)$ y menor entre los viudos (7,4\%) (Tabla 2).

La Tabla 3 presenta la razón de prevalencia (bruta y ajustada) de los comportamientos de riesgo relacionados con hombres mayores de acuerdo a su situación conyugal. Los viudos fueron considerados como el grupo de referencia. De los cuatro comportamientos investigados solo en el tabaquismo se observó

Tabla 2. Prevalencia de los comportamientos de riesgos para la salud en hombres mayores del sexo masculino, según situación conyugal (general y estratificado por rango de edad y escolaridad), residentes en las capitales y Distrito Federal, Vigitel, Brasil, 2016 y $2017(n=11.185)$.

\begin{tabular}{|c|c|c|c|c|c|c|c|c|c|c|c|c|}
\hline \multirow[t]{2}{*}{ Variables } & \multicolumn{2}{|c|}{ IFTL } & \multirow[t]{2}{*}{ Valor de $p$} & \multicolumn{2}{|c|}{ CIFVL } & \multirow[t]{2}{*}{ Valor de $p$} & \multicolumn{2}{|c|}{$\mathrm{TAB}$} & \multirow[t]{2}{*}{ Valor de $p$} & \multicolumn{2}{|c|}{ CAA } & \multirow[t]{2}{*}{ Valor de $p$} \\
\hline & $n$ & $\%$ & & $n$ & $\%$ & & $\mathrm{n}$ & $\%$ & & $n$ & $\%$ & \\
\hline Viudo & 627 & 70,8 & \multirow{4}{*}{0,175} & 525 & 59,3 & \multirow{4}{*}{0,186} & 77 & 8,7 & \multirow{4}{*}{$<0,001$} & 73 & 8,2 & \multirow{4}{*}{$<0,001$} \\
\hline Con pareja & 6.123 & 69,7 & & 5.003 & 56,9 & & 678 & 7,7 & & 1.151 & 13,1 & \\
\hline Divorciado/Separado & 566 & 67,4 & & 503 & 59,9 & & 119 & 14,2 & & 127 & 15,1 & \\
\hline Soltero & 450 & 66,8 & & 398 & 59,1 & & 95 & 14,1 & & 101 & 15,0 & \\
\hline \multicolumn{13}{|l|}{$\begin{array}{l}\text { Rango de edad } \\
60 \text { a } 69 \text { años }\end{array}$} \\
\hline Viudo & 155 & 69,2 & \multirow{4}{*}{0,462} & 145 & 64,7 & \multirow{4}{*}{0,289} & 26 & 11,6 & \multirow{4}{*}{$<0,001$} & 32 & 14,3 & \multirow{4}{*}{0,415} \\
\hline Con pareja & 3.426 & 68,7 & & 2.945 & 59,0 & & 476 & 9,5 & & 879 & 17,6 & \\
\hline Divorciado/Separado & 348 & 68,4 & & 301 & 59,1 & & 91 & 17,9 & & 98 & 19,3 & \\
\hline Soltero & 292 & 65,0 & & 276 & 61,5 & & 72 & 16,0 & & 83 & 18,5 & \\
\hline \multicolumn{13}{|l|}{70 a 79 años } \\
\hline Viudo & 239 & 69,1 & & 216 & 62,4 & & 39 & 11,3 & & 30 & 8,7 & \\
\hline Con pareja & 2.015 & 69,2 & & 1.603 & 55,1 & & 166 & 5,7 & & 257 & 8,8 & \\
\hline Divorciado/Separado & 173 & 67,3 & 0,919 & 158 & 61,5 & 0,019 & 27 & 10,5 & $<0,001$ & 25 & 9,7 & 0,920 \\
\hline Soltero & 127 & 70,2 & & 100 & 55,2 & & 21 & 11,6 & & 18 & 9,9 & \\
\hline 80 años o más & & & & & & & & & & & & \\
\hline Viudo & 233 & 73,7 & & 164 & 51,9 & & 12 & 3,8 & & 11 & 3,5 & \\
\hline Con pareja & 682 & 77,1 & & 455 & 51,5 & & 36 & 4,1 & & 15 & 1,7 & \\
\hline Divorciado/Separado & 45 & 60,8 & 0,012 & 44 & 59,5 & 0,611 & 1 & 1,4 & $0, / 01$ & 4 & 5,4 & 0,053 \\
\hline Soltero & 31 & 70,5 & & 22 & 50,0 & & 2 & 4,5 & & 0 & 0,0 & \\
\hline Escolaridad & & & & & & & & & & & & \\
\hline 0 a 8 años & & & & & & & & & & & & \\
\hline Viudo & 345 & 74,4 & & 315 & 67,9 & & 44 & 9,5 & & 25 & 5,4 & \\
\hline Con pareja & 2.419 & 77,4 & & 2.206 & 70,6 & & 303 & 9,7 & & 279 & 8,9 & \\
\hline Divorciado/Separado & 223 & 75,3 & $0,01 /$ & 215 & 72,6 & 0,386 & 42 & 14,2 & 0,089 & 30 & 10,1 & 0,042 \\
\hline Soltero & 160 & 69,0 & & 170 & 73,3 & & 26 & 11,2 & & 24 & 10,3 & \\
\hline 9 a 11 años & & & & & & & & & & & & \\
\hline Viudo & 138 & 71,5 & & 112 & 58,0 & & 18 & 9,3 & & 20 & 10,4 & \\
\hline Con pareja & 1.662 & 69,9 & & 1.469 & 61,8 & & 169 & 7,1 & & 356 & 15,0 & \\
\hline Divorciado/Separado & 160 & 66,7 & 0,591 & 158 & 65,8 & 0,405 & 33 & 13,8 & $<0,001$ & 39 & 16,3 & 0,303 \\
\hline Soltero & 150 & 72,1 & & 131 & 63,0 & & 30 & 14,4 & & 33 & 15,9 & \\
\hline 12 años o más & & & & & & & & & & & & \\
\hline Viudo & 144 & 62,9 & & 98 & 42,8 & & 15 & 6,6 & & 28 & 12,2 & \\
\hline Con pareja & 2.042 & 62,2 & 0708 & 1.328 & 40,5 & 0785 & 206 & 6,3 & & 516 & 15,7 & \\
\hline Divorciado/separado & 183 & 60,2 & 0,198 & 130 & 42,8 & $0, / 85$ & 44 & 14,5 & $<0,001$ & 58 & 19,1 & 0,105 \\
\hline Soltero & 140 & 59,8 & & 97 & 41,5 & & 39 & 16,7 & & 44 & 18,8 & \\
\hline Raza/Color & & & & & & & & & & & & \\
\hline Blanco/Amarillo & & & & & & & & & & & & \\
\hline Viudo & 378 & 69,6 & & 300 & 55,2 & & 46 & 8,5 & & 40 & 7,4 & \\
\hline Con pareja & 3.454 & 69,4 & & 2.520 & 50,6 & & 381 & 7,7 & & 610 & 12,3 & \\
\hline Divorciado/Separado & 335 & 67,5 & 0,438 & 277 & 55,8 & 0,039 & 75 & 15,1 & $<0,001$ & 70 & 14,1 & 0,003 \\
\hline Soltero & 242 & 65,8 & & 188 & 51,1 & & 61 & 16,6 & & 47 & 12,8 & \\
\hline Preto/Pardo/Indígena & & & & & & & & & & & & \\
\hline Viudo & 54 & 75,0 & & 47 & 65,3 & & 10 & 13,9 & & 6 & 8,3 & \\
\hline Con pareja & 427 & 73,5 & & 408 & 70,2 & 0647 & 44 & 7,6 & & 81 & 13,9 & 0677 \\
\hline Divorciado/Separado & 39 & 65,0 & 0,540 & 44 & 73,3 & 0,641 & 6 & 10,0 & 0,159 & 8 & 13,3 & 0,621 \\
\hline Soltero & 43 & 72,9 & & 44 & 74,6 & & 8 & 13,6 & & 8 & 13,6 & \\
\hline
\end{tabular}

Fuente: Sistema de Vigilancia de Factores de Riesgo y Protección para Enfermedades Crónicas por Encuesta Telefónica (VIGITEL) 2016 y 2017, Ministerio de Salud, Brasil.

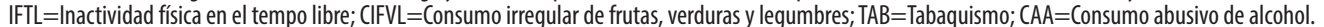


Tabla 3. Razón de prevalencia de los comportamientos de riesgo relacionados a la salud en hombres mayores residentes en las capitales brasileñas y Distrito Federal, según situación conyugal, Vigitel, Brasil, 2016 y 2017 (n=11.185).

\begin{tabular}{|c|c|c|c|c|c|c|c|c|c|c|c|c|c|c|c|c|}
\hline \multirow[t]{2}{*}{ Variables } & \multicolumn{4}{|c|}{ Inactividad física en el tiempo libre } & \multicolumn{4}{|c|}{$\begin{array}{l}\text { Consumo irregular de frutas, verduras y } \\
\text { legumbres }\end{array}$} & \multicolumn{4}{|c|}{ Tabaquismo } & \multicolumn{4}{|c|}{ Consumo abusivo de alcohol } \\
\hline & $\begin{array}{l}\mathrm{RP} \\
\text { bruta }\end{array}$ & IC95\% & $\begin{array}{c}\mathrm{RP} \\
\text { ajustada' }\end{array}$ & IC95\% & $\begin{array}{c}\mathrm{RP} \\
\text { bruta }\end{array}$ & IC95\% & $\begin{array}{c}\mathrm{RP} \\
\text { ajustada' }\end{array}$ & IC95\% & $\underset{\text { bruta }}{\mathrm{RP}}$ & IC95\% & $\begin{array}{l}\mathrm{RP} \\
\text { ajustada' }\end{array}$ & IC95\% & $\begin{array}{c}\mathrm{RP} \\
\text { bruta }\end{array}$ & IC95\% & $\begin{array}{c}\mathrm{RP} \\
\text { ajustada' }\end{array}$ & IC95\% \\
\hline Viudo & 1,00 & & 1,00 & & 1,00 & & 1,00 & & 1,00 & & 1,00 & & 1,00 & & 1,00 & \\
\hline Con pareja & 0,98 & $0,94-1,03$ & 1,04 & $0,98-1,10$ & 0,96 & $0,91-1,02$ & 0,98 & $0,91-1,05$ & 0,89 & $0,71-1,11$ & 0,68 & $0,52-0,90$ & 1,59 & $1,27-1,99$ & 1,08 & $0,81-1,44$ \\
\hline $\begin{array}{l}\text { Divorciado/ } \\
\text { Separado }\end{array}$ & 0,95 & $0,89-1,01$ & 1,00 & $0,92-1,08$ & 1,01 & $0,93-1,09$ & 1,07 & $0,97-1,18$ & 1,63 & $1,24-2,14$ & 1,25 & $0,91-1,73$ & 1,83 & $1,40-2,41$ & 1,16 & $0,82-1,63$ \\
\hline Soltero & 0,94 & $0,88-1,01$ & 0,99 & $0,91-1,08$ & 1,00 & $0,92-1,08$ & 0,98 & $0,88-1,10$ & 1,62 & $1,22-2,15$ & 1,31 & $0,93-1,84$ & 1,82 & $1,37-2,42$ & 1,00 & $0,69-1,45$ \\
\hline
\end{tabular}

Fuente: Sistema de Vigilancia de Factores de Riesgo y Protección para Enfermedades Crónicas por Encuesta Telefónica (Vigitel) 2016 y 2017, Ministerio de Salud, Brasil.

${ }^{1}$ Razón de prevalencia ajustada por escolaridad, rango de edad y raza/color.

$\mathrm{RP}=$ Razón de prevalencia. IC95\%= Intervalo de confianza del 95\%.

asociación, siendo menor entre individuos con pareja $[R P=0,68 ; \mathrm{IC} 95 \%(0,52-0,90)]$ que entre los viudos.

Se realizaron también análisis estratificados por edad, escolaridad y raza/color no presentados en la tabla, ajustándose por las demás variables demográficas, excepto por aquella que era la variable independiente. En este análisis, se observó una menor prevalencia de tabaquismo en los hombres de 70 a 79 años y en aquellos con pareja $[R P=0,52$; IC95\% (0,35-0,77)]. En los hombres que tenían de 9 a 11 años de estudio también se observó menor prevalencia entre los que tenían pareja $[R P=0,52 ; \operatorname{IC95\% }(0,31-0,87)]$. Ya entre los de mayor escolaridad (12 años o más) la prevalencia del tabaquismo fue mayor que los solteros [RP =2,23; IC95\% $(1,21-4,13)]$. Se observó también, entre negros, pardos o indígenas, una mayor prevalencia de consumo irregular de frutas, verduras y legumbres entre divorciados/separados $[R P=1,27 ; \mathrm{IC} 95 \%$ $(1,02-1,58)]$ que entre los viudos (dato no presentado en tablas).

Tabla 4. Asociación entre rango de edad, escolaridad y raza/color con comportamientos de riesgo a la salud en hombres mayores residentes en las capitales brasileñas y Distrito Federal, según rango de edad, escolaridad y raza/color, Vigitel, 2016 y 2017 (n=886).

\begin{tabular}{|c|c|c|c|c|c|c|c|c|c|c|c|c|c|c|c|c|}
\hline \multirow[t]{2}{*}{ Variables } & \multicolumn{4}{|c|}{ IFTL } & \multicolumn{4}{|c|}{ CIFVL } & \multicolumn{4}{|c|}{$\mathrm{TAB}$} & \multicolumn{4}{|c|}{ CAA } \\
\hline & $\begin{array}{c}\mathrm{RP} \\
\text { bruta }\end{array}$ & IC95\% & $\begin{array}{c}\mathrm{RP} \\
\text { ajustada }\end{array}$ & IC95\% & $\begin{array}{c}\mathrm{RP} \\
\text { bruta }\end{array}$ & IC95\% & $\begin{array}{c}\text { RP } \\
\text { ajustada }\end{array}$ & IC95\% & $\begin{array}{c}\mathrm{RP} \\
\text { bruta }\end{array}$ & IC95\% & $\begin{array}{c}\mathrm{RP} \\
\text { ajustada }\end{array}$ & IC95\% & $\begin{array}{c}\text { RP } \\
\text { bruta }\end{array}$ & IC95\% & $\begin{array}{c}\mathrm{RP} \\
\text { ajustada }\end{array}$ & IC95\% \\
\hline \multicolumn{17}{|l|}{ Rango de edad' } \\
\hline 60 a 69 años & 1,00 & & 1,00 & & 1,00 & & 1,00 & & 1,00 & & 1,00 & & 1,00 & & 1,00 & \\
\hline 70 a 79 años & 1,00 & $0,89-1,12$ & 0,86 & $0,74-0,98$ & 0,96 & $0,85-1,10$ & 0,93 & $0,79-1,10$ & 0,97 & $0,61-1,55$ & 1,01 & $0,56-1,80$ & 0,61 & $0,38-0,97$ & 1,06 & $0,57-1,98$ \\
\hline 80 anõs o más & 1,07 & $0,95-1,19$ & 0,97 & $0,86-1,10$ & 0,80 & $0,69-0,93$ & 0,78 & $0,66-0,93$ & 0,33 & $0,17-0,63$ & 0,32 & $0,15-0,65$ & 0,24 & $0,13-0,47$ & 0,35 & $0,15-0,83$ \\
\hline \multicolumn{17}{|l|}{ Escolaridad $^{2}$} \\
\hline 12 años o más & 1,00 & & 1,00 & & 1,00 & & 1,00 & & 1,00 & & 1,00 & & 1,00 & & 1,00 & \\
\hline 9 a 11 años & 1,14 & $0,99-1,30$ & 1,16 & $1,00-1,35$ & 1,36 & $1,12-1,64$ & 1,31 & $1,04-1,65$ & 1,42 & $0,74-2,75$ & 1,51 & $0,73-3,11$ & 0,85 & $0,49-1,46$ & 0,91 & $0,48-1,74$ \\
\hline 0 a 8 años & 1,18 & $1,06-1,32$ & 1,15 & $1,00-1,31$ & 1,59 & $1,35-1,87$ & 1,60 & $1,31-1,94$ & 1,45 & $0,82-2,54$ & 1,30 & $0,68-2,49$ & 0,44 & $0,26-0,74$ & 0,36 & $0,17-0,76$ \\
\hline \multicolumn{17}{|l|}{ Raza/color ${ }^{3}$} \\
\hline Blanco/amarillo & 1,00 & & 1,00 & & 1,00 & & 1,00 & & 1,00 & & 1,00 & & 1,00 & & 1,00 & \\
\hline $\begin{array}{l}\text { Negro/pardo/ } \\
\text { indígena }\end{array}$ & 1,08 & $0,93-1,24$ & 1,04 & $0,90-1,20$ & 1,18 & $0,98-1,42$ & 1,00 & $0,83-1,21$ & 1,64 & $0,87-3,10$ & 1,32 & $0,67-2,60$ & 1,13 & $0,50-2,57$ & 1,40 & $0,56-3,47$ \\
\hline
\end{tabular}

Fuente: Sistema de Vigilancia de Factores de Riesgo y Protección para Enfermedades Crónicas por Encuesta Telefónica (Vigitel) 2016 y 2017, Ministerio de Salud, Brasil.

${ }^{1}$ Ajustado por escolaridad y raza/color; ${ }^{2}$ Ajustado por rango de edad y raza/color; ${ }^{3}$ jjustado por rango de edad y escolaridad.

IFTL=Inactividad física en el tempo libre; $C I F V L=C o n s u m o$ irregular de frutas, verduras y legumbres; TAB=Tabaquismo; $C A A=C o n s u m o$ abusivo de alcohol. 
En los otros comportamientos no se observaron asociaciones significativas. Sin embargo, el extremo inferior del intervalo de confianza estuvo al límite de asumir una diferencia significativa en el caso de la inactividad física en el tiempo libre entre aquellos con 70 a 79 años con pareja $[R P=1,11$; IC95\% $(1,00-$ $1,23)$ ] y entre aquellos con escolaridad igual o menor a ocho años, también con pareja $[R P=1,07$; IC95\% $(1,00-1,16)]$ (datos no mostrados en las tablas).

La Tabla 4 presenta las razones de prevalencia de la asociación entre edad, escolaridad y raza/color y los comportamientos de hombres viudos. Con relación a la inactividad física en el tiempo libre, se observó una menor prevalencia entre viudos del rango de edad intermedia, entre 70 y 79 años de edad [RP $=0,86$; IC95\% $(0,74-0,98)]$. Con relación a la escolaridad, el valor observado de la razón de prevalencia ajustada fue de 1,16 para aquellos con escolaridad intermedia (9 a 11 años) y de 1,15 para los hombres con menor escolaridad $(0 \mathrm{a}$ 8 años), mientras el valor inferior del intervalo de confianza se dio en el límite entre la asociación y la no asociación entre ambos casos (valor igual a 1,00). El consumo irregular de frutas, verduras y legumbres fue inferior entre los viudos con edad igual o mayor de 80 años $[R P=1,60 ; \operatorname{IC} 95 \%(0,66-0,93)]$ y mayor entre los que tenían hasta ocho años de escolaridad $[R P=1,60 ; \operatorname{IC} 95 \%(1,31-1,94)]$ y de 9 a 11 años $[R P=1,31$; IC95\% $(1,04-1,65)]$ en comparación con el grupo de referencia (12 años o más de escolaridad). Respecto al tabaquismo, se observó menor prevalencia entre los viudos de mayor edad $[R P=0,32$; IC95\% $(0,15-0,65)]$ lo mismo sucedió con el consumo abusivo de alcohol $[R P=0,35 ; \operatorname{IC} 95 \%(0,15-0,83)]$. Se observó también menor prevalencia entre aquellos con menor escolaridad $[\mathrm{RP}=0,36$; IC95\% $(0,17-0,76)]$.

\section{DISCUSIÓN}

$\mathrm{Al}$ analizar las prevalencias de los comportamientos de riesgo para la salud según la situación conyugal, se encontraron asociaciones importantes; sin embargo, al comparar las diferencias entre los viudos con otros grupos, solo se observó una menor prevalencia de fumadores en aquellos con pareja que entre viudos. Cuando el análisis fue hecho por edad, escolaridad y raza/color, hubo pocas diferencias entre los viudos y otros grupos. Al comparar las prevalencias de los comportamientos de riesgo entre los hombres viudos, según la edad, la escolaridad y la raza/color, se observaron asociaciones importantes con la edad (en todos los comportamientos investigados) y escolaridad (consumo irregular de frutas, abuso de vegetales y alcohol). Por otro lado, no se encontraron asociaciones significativas con la raza/color.

Se observaron diferencias en las prevalencias de los comportamientos de riesgo según la situación conyugal. Estas diferencias son coherentes con estudios anteriores ${ }^{(22,25,26,27)}$. Diversos factores pueden explicar estas diferencias. Por una parte, si estar casado o en unión estable puede relacionarse con la adopción de comportamientos considerados saludables, debido a una red de apoyo social más grande, por otra parte, una calidad de la relación considerada mala puede hacer que el sujeto adopte comportamientos de riesgo ${ }^{(32)}$.

A diferencia del tabaquismo, se observaron asociaciones tímidas de los comportamientos de riesgo en la comparación entre la viudez con las demás situaciones conyugales. En general, la viudez aparece asociada a peores indicadores de salud, que pueden estar relacionados con factores estresantes como: muerte de la pareja, la pérdida de soporte y apoyo social, la necesidad de recomenzar la vida y de rehacer lazos sociales, siendo todo eso, más difícil para adultos mayores ${ }^{(1,14,15,33)}$. Por otra parte, se puede relacionar la viudez con un recomienzo o inicio de una nueva vida con mayor libertad ${ }^{(17)}$.

Como ya fue mencionado, se observó menor prevalencia de tabaquismo en hombres con pareja. Estudios indican resultados semejantes con relación a los sujetos casados o en unión estable $\mathrm{e}^{(14,24,34,35,36)}$. Una explicación posible es la ya mencionada protección del casamiento, que se relaciona a una mayor red de apoyo social, soporte económico 
y psicológico. Más allá de eso, tiene relación con la cuestión de género. En general, el hombre se beneficia más del cuidado a la salud y el soporte/apoyo social otorgado por la compañera y, la ausencia de este cuidado, puede favorecer la adopción de comportamientos de riesgo ${ }^{(14,17,24,33)}$.

En comparación con los hombres de 60 a 69 años, hubo una menor prevalencia de la inactividad física en el tiempo libre en los hombres de 70 a 79. A diferencia de este resultado, estudios previos que verificaron los niveles de actividad física en el tiempo libre en los adultos mayores identificaron que la prevalencia aumenta con la edad ${ }^{(37,38)}$, aunque estos estudios no realizaron análisis estratificados de acuerdo con la situación conyugal de los adultos mayores. Una posible razón para el resultado encontrado en el presente estudio puede deberse al sesgo de supervivencia, dado que la inactividad física está asociada con la mortalidad, es posible que muchas personas de edad avanzada que murieron antes de cumplir 70 años hayan estado en promedio menos activas físicamente que los que alcanzaron esta $\operatorname{edad}^{(39,40,41)}$. Otra posible explicación puede ser el hecho de que probablemente haya una mayor proporción de jubilados entre aquellos con 70 a 79 años que entre los con 60 a 69 años, y es plausible imaginar que los jubilados tengan más tiempo libre y puedan ser más activos en este dominio que las personas no jubiladas. Por supuesto, se debe tener precaución con estos hallazgos, y se necesitan más estudios, incluso para confirmar si hay alguna tendencia diferente entre los hombres viudos que en aquellos con otras situaciones conyugales con respecto a la actividad física en el tiempo libre a medida que envejecen y, si se observan diferencias, los estudios deben buscar dilucidar las posibles razones de esta especificidad.

En cuanto a la escolaridad, hubo una mayor prevalencia de inactividad física en el tiempo libre entre los hombres con educación intermedia (9 a 11 años de escolaridad) y baja (0 a 8 años de escolaridad), en comparación a aquellos con educación superior ( $\geq 12$ años de estudio). Estos resultados indican que la relación entre la escolaridad y la actividad física en el tiempo libre en los hombres viudos es similar a la de la población general, ya que hay mucha evidencia de que los sujetos con más años de escolaridad tienden a ser más activos que aquellos con niveles más bajos de escolaridad ${ }^{(42,43,44,45)}$.

Los viudos de edad avanzada ( $\geq 80$ años) tuvieron un menor consumo irregular de frutas, verduras y legumbres en comparación con los viudos más jóvenes (60 a 69 años). Los estudios indican que este mayor consumo en los hombres de edad avanzada está asociado con la prevención de enfermedades crónicas y una menor mortalidad ${ }^{(46,47)}$. Un factor que puede explicar este hallazgo es también el sesgo de supervivencia. Puede ser que los sujetos que consumieron más frutas, verduras y legumbres irregulares hayan muerto antes. Además, las personas mayores pueden tener una preocupación más inmediata por su salud, así como más tiempo para preparar y comprar estos alimentos, ya que es probable que una mayor proporción de personas del grupo más joven de este estudio, todavía estén trabajando. Sin embargo, como se trata de un estudio transversal, no es posible establecer una relación causal para saber si los hombres más viejos ( $\geq 80$ años) cambiaron su comportamiento o mantuvieron el mismo estilo de vida que cuando tenían 60 años.

También hubo una mayor prevalencia de consumo irregular de frutas, verduras $y$ legumbres en sujetos con niveles educativos más bajos (0 a 8 y 9 a 11 años) una asociación lineal, lo que indica una mayor desventaja entre aquellos con educación inferior (0 a 8 años). En general, los estudios analizados asocian la menor escolaridad con el bajo consumo de frutas, verduras y legumbres $^{(48,49,50,51,52,53)}$. Según Silveira et al. ${ }^{(50)}$, la educación está relacionada con un mejor conocimiento, que favorece la adopción de mejores comportamientos relacionados con la salud, los sujetos que tienen un nivel de educación superior tienen más probabilidades de estar mejor informados sobre la adopción de comportamientos saludables, porque les resulta más fácil buscar y comprender información para mantener comportamientos saludables o cambiar comportamientos 
de riesgo. Además, en general, la educación superior está asociada con un mayor poder adquisitivo. En el contexto brasileño, la barrera de costos en la adquisición de estos productos parece ser un factor importante. Santos et al. ${ }^{(48)}$ realizaron un estudio transversal que verificó las principales barreras para el consumo de frutas, verduras y legumbres y descubrió que los sujetos con hasta cuatro años de escolaridad tenían más probabilidades de referirse a la "carga de costos en el presupuesto familiar" para comprar frutas, verduras y legumbres.

Al comparar la prevalencia del tabaquismo en los hombres viudos según las variables demográficas, se observó una menor prevalencia en los hombres más viejos ( $\geq 80$ años). Este resultado también puede estar relacionado con el sesgo de supervivencia, ya que existe evidencia clara en la bibliografía que indica la relación entre el tabaquismo y la mortalidad ${ }^{(54,55,56,57,58)}$. Para Zaitune et al..$^{(54)}$, la menor prevalencia observada en los adultos mayores puede explicarse por la aparición temprana de enfermedades asociadas con el tabaquismo que conducen a la recomendación de dejar de fumar, una mayor probabilidad de muerte prematura y la creciente preocupación por su propia salud, lo que hace que las personas mayores adopten comportamientos más saludables. En este sentido, realizamos un análisis adicional (datos no mostrados) en el que se observó que la proporción de exfumadores entre los viudos de nuestra muestra era mayor a medida que aumentaba el grupo de edad $(52,2 \%$ de 60 a 69 años, $56,6 \%$ de 70 y 79 años, y $62,0 \%$ en aquellos con 80 años o más).

El menor consumo abusivo de alcohol fue observado entre los hombres viudos con escolaridad más baja. Estudios previos muestran que personas con peor nivel socioeconómico son más vulnerables a los problemas y consecuencias del consumo abusivo de alcohol. Además, al igual que con otros comportamientos de riesgo, puede haber un sesgo de supervivencia en esos hombres mayores.

Entre las limitaciones del estudio se encuentra el diseño transversal que hace que sea imposible establecer una relación causal entre las variables. Además, la representatividad limitada a las 26 capitales estatales de Brasil y el Distrito Federal, además del muestreo por telefonía fija, puede afectar la precisión de la información sobre los comportamientos, especialmente, respecto a la posible dificultad que algunas personas mayores pueden tener para responder preguntas por teléfono.

Por otro lado, entre los puntos positivos, es digno de mención que, posiblemente, este sea un estudio sin precedentes con este tema en el contexto latinoamericano. Además, investigamos la prevalencia de cinco comportamientos en una muestra representativa de hombres mayores de todas las capitales estatales y del Distrito Federal de Brasil.

Los resultados muestran la complejidad del tema y la necesidad de que se realicen otros estudios, como los longitudinales que señalan el impacto de la viudez en la adopción de comportamientos positivos y de riesgo para la salud. También se sugiere que estos estudios busquen medir aspectos que puedan mediar la relación entre la viudez y los comportamientos, como el tema del apoyo social. Los estudios cualitativos que buscan una comprensión más profunda del fenómeno también pueden ser de gran importancia.

Este estudio mostró asociaciones moderadas entre los comportamientos de riesgo de la viudez en hombres mayores, en las que solo había una menor prevalencia de tabaquismo entre aquellos con pareja en comparación con los viudos. Por otro lado, considerando solo a los hombres mayores viudos, se observaron asociaciones de acuerdo con la edad y escolaridad, pero no con la raza/color. Dado este contexto, es importante que los profesionales de la salud tengan una visión más amplia de la atención, así como una visión más humanizada de los diversos factores que pueden contribuir al proceso de atención de la salud y la enfermedad de los hombres, especialmente, los mayores. Por lo tanto, este estudio puede ser importante para planificar acciones dirigidas a promover comportamientos saludables en la salud de los hombres viudos. 


\section{REFERENCIAS BIBLIOGRÁFICAS}

1. Fernandes $B L$, Borgato $M H$. A viuvez e a saúde dos idosos: uma revisão integrativa. Revista Kairós Gerontologia. 2016;19(3):187-204.

2. Camarano AA. Mulher idosa: suporte familiar ou agente de mudança? Estudos Avançados. 2003;17(49):35-63.

3. Cruz MF, Ramires VV, Wendt A, Mielke GI, Martinez-Mesa J, Wehrmeister FC. Simultaneidade de fatores de risco para doenças crônicas não transmissíveis entre idosos da zona urbana de Pelotas, Rio Grande do Sul, Brasil. Cadernos de Saúde Pública. 2017;33(2):e00021916.

4. Vasconcelos A, Gomes M. Transição demográfica: a experiência brasileira. Epidemiologia e Serviços de Saúde. 2012;21:539-548.

5. Francisco PMSB, Segri NJ, Borim FSA, Malta DC. Prevalência simultânea de hipertensão e diabetes em idosos brasileiros: desigualdades individuais e contextuais. Ciência e Saúde Coletiva. 2018;23(11):3829-3840.

6. Barata RB. Como e por que as desigualdades sociais fazem mal à saúde. Rio de Janeiro: Fiocruz; 2009.

7. Carr D, Bodnar-Deren S. Gender, aging and widowhood. In: Uhlenberg P. International handbook of population aging. Dordrecht: Springer; 2009.

8. Gomes R, Nascimento EF. A produção do conhecimento da saúde pública sobre a relação homem-saúde: uma revisão bibliográfica. Cadernos de Saúde Pública. 2006;22(5)901-911.

9. Gomes R, Martha Cristina Nunes Moreira MCN, Nascimento EF, Rebello LEFS, Couto MT, Schraiber LB. Os homens não vêm! Ausência e/ ou invisibilidade masculina na atenção primária. Ciências e Saúde Coletiva. 2011;16(Supl 1):983992.

10. Brasil. Ministério da Saúde(MS). Política nacional de atenção integral à saúde do homem. Brasília: MS; 2009.

11. Manzoli L, Villari P, Pirone GM, Boccia A. Marital status and mortality in the elderly: A systematic review and meta-analysis. Social Science and Medicine. 2007;64:77-94.

12. Eng PM, Kawachi I, Fitzmaurice G, Rimm EB. Effects of marital transitions on changes in dietary and other health behaviours in US male health professionals. Journal of Epidemiology and Community Health. 2005;59(1):56-62.
13. Gomes MMF, Turra CM, Fígoli MGB, Duarte YAO, Lebrão ML. Associação entre mortalidade e estado marital: uma análise para idosos residentes no Município de São Paulo, Brasil, Estudo SABE, 2000 e 2006. Cadernos de Saúde Pública. 2013;29(3):566-578.

14. Umberson D. Gender, marital status and the social control of health behavior. Social Science \& Medicine. 1992;34(8):907-917.

15. Williams K, Umberson D. Marital status, marital transitions and health: a gendered life course perspective. Journal of Health \& Social Behavior. 2004;45(1):81-98

16. Gomes R. A Saúde do Homem em Foco. São Paulo: Unesp; 2010.

17. Tôrres EM. A viuvez na vida dos idosos [Dissertação de mestrado]. Salvador: Universidade Federal da Bahia; 2006.

18. Colombo VXL, Gatto MRT, Aristizábal-Vallejo $\mathrm{N}$, Angarita RB, Calderón DH, Miranda LMM, Guiñazu NP, Gutiérrez LMT, Tarifa EC, Palacios M, Campos CIV. Viúdez y vejez en América Latina. Kairós Gerontologia. 2014;17(1):9-26.

19. Motta AB. Sociabilidades possíveis: idosos e tempo geracional. En: Peixoto CE. Família e envelhecimento. Rio de Janeiro: Editora FGV; 2004.

20. Ho SH, Hung WS. The impacts of widowhood, chronic disease and physical function on mortality among older people. Journal of Nursing Research. 2013;21(2):110-119.

21. Stimpson JP, Kuo YK, Ray LA, Raji MA, Peek $K$. Risk of mortality related to widowhood in older mexican americans. Annals of Epidemiology. 2007; 17(4):313-319.

22. Williams K. The transition to widowhood and the social regulation of health: consequences for health and health risk behavior. Journal of Gerontology. 2004;59(6):343-349.

23. Skulason B, Jonsdottir LS, Sigurdardottir V, Helgason AR. Assessing survival in widowers and controls: A nationwide six-to nine-year follow-up. BMC Public Health. 2012;12(96):1-8.

24. Perkins JM, Lee HY, Lee JK, Heo J, Krishna A, Choi S, Nam YS, Oh J, Subramanian SV. Widowhood and alcohol, tobacco, and other drug use among older adults in India. Gerontological Society of América. 2016;0(0)1-9.

25. Eng PM, Kawachi I, Fitzmaurice G, Rimm EB. Effects of marital transitions on changes in dietary and other health behaviours in US male health 
professionals. Journal of Epidemiology and Community Health. 2005;59(1):56-62.

26. Vinther JL, Conklin AJ, Wareham NJ, Monsivais P.Marital transitions and associated changes in fruit and vegetable intake: Findings from the populationbased prospective EPIC-Norfolk cohort, UK. Social Science and Medicine. 2016;157:120-126.

27. Francisco PMSB, Segri NJ, Borim FSA, Malta DC. Prevalência simultânea de hipertensão e diabetes em idosos brasileiros: desigualdades individuais e contextuais. Ciência e Saúde Coletiva. 2018;23(11):3829-3840.

28. Rolden HJA, van Bodegom D, Westendorp RGJ. Changes in health care expenditure after the loss of a spouse: data on 6.487 older widows and widowers in the Netherlands. PLoS One. 2014; 9(12):1-17.

29. Ministério da Saúde. Vigilância de fatores de risco e proteção para doenças crônicas por inquérito telefônico 2016. Brasília: MS; 2017.

30. Ministério da Saúde. Vigilância de fatores de risco e proteção para doenças crônicas por inquérito telefônico 2017. Brasília: MS; 2018.

31. World Health Organization. Global recommendations on physical activity for health [Internet]. Geneva: WHO; 2011 [citado 9 dic 2019]. Disponible en: https://tinyurl.com/ufrnu5c.

32. Robles TF. Marital quality and health: Implications for marriage in the 21st century. Current Directions in Psychological Science. 2014;23(6):427-433.

33. Ho SH. A comparative assessment of emergency medicine between the widowers and widows among the elderly in Taiwan. Economics Bulletin. 2015;35(3):1795-1808.

34. Cho HJ, Khang YH, Jun HJ, Kawachi I. Marital status and smoking in Korea: The influence of gender and age. Social Science and Medicine. 2008;(66):609-619.

35. Nystadt P. Marital life course events and smoking behaviour in Sweden 1980-2000. Social Science \& Medicine. 2006;62(6):1427-1442.

36. Rhakonen $\mathrm{O}$, Laaksonen $\mathrm{M}$, Karvonen S. The contribution of lone parenthood and economic difficulties to smoking. Social Science \& Medicine. 2005;61(1):211-216.

37. Bueno DR, Marucci MFN, Roediger MA, Gomes IC, Duarte YAO, Lebrão ML. Nível de atividade física, por acelerometria, em idosos do município de São Paulo: Estudo SABE. Revista Brasileira de Medicina do Esporte. 2010;22(2):108112.
38. Mourão ARC, Novais FV, Andreoni S, Ramos LR. Atividade física de idosos relacionada ao transporte e lazer, Maceió, Brasil. Revista de Saúde Pública. 2013;47(6):1112-1122.

39. Katzmarzyk PT. Effect of physical inactivity on major non-communicable diseases worldwide: an analysis of burden of disease and life expectancy. The Lancet. 2012;380(9838):219-229.

40. Rezende LFM, Rabacow FM, Viscondi JYK, Luiz OC, Matsudo VKR, Lee IM. Effect of physical inactivity on major noncommunicable diseases and life expectancy in Brazil. Journal of Physical Activity and Health. 2015;12(3):299-306.

41. Silva DAS, Malta DC, Souza MFM, Naghavi $M$. Burden of ischemic heart disease mortality attributable to physical inactivity in Brazil. Revista de Saúde Pública. 2018;52(72):1-10.

42. Rodrigues PAF, Melo MP, Assis MR, Palma A. Condições socioeconômicas e prática de atividades físicas em adultos e idosos: uma revisão sistemática. Revista Brasileira de Atividade Física e Saúde. 2017;22(3):217-232.

43. Forechi L, Mill JG, Griep RH, Santos I, Pitanga F, Molina MCB. Adherence to physical activity in adults with chronic diseases: ELSA-Brasil. Revista de Saúde Pública. 2017;52(31):1-12.

44. Aro $\mathrm{AA}, \mathrm{Agbo} \mathrm{S}$, Omole OB. Factors influencing regular physical exercise among the elderly in residential care facilities in a South African health district. African Journal of Primary Health Care and Family. 2018;10(1):1-6.

45.Peixoto SV, Mambrini JVM, Firmo JOA, Loyola AI, Souza PRB, Andrade FB, Lima-Costa MF. Prática de atividade física entre adultos mais velhos: resultados do ELSI-Brasil. Revista de Saúde Pública. 2018;52(Supl 2):1-9.

46. Wang L, Manson JE, Gaziano JM, Buring JE, Sesso HD. Fruit and vegetable intake and the risk of hypertension in middle-aged and older women. American Journal of Hypertension. 2012;25(2): 180-189.

47. Nicklett EJ, Kadell AR. Fruit and vegetable intake among older adults: a scoping review. Maturitas. 2013;75(4):305-312.

48. Santos GMGC, Silva AMR, Loch MR, Carvalho W, Rech CR. Barreiras percebidas para o consumo de frutas e de verduras ou legumes em adultos brasileiros. Ciência \& Saúde Coletiva. 2019;24(7): 2461-2470.

49. Jaime PC, Figueiredo ICR, Moura EC, Malta DC. Fatores associados ao consumo de frutas e 
hortaliças no Brasil, 2006. Revista de Saúde Pública. 2009;43(Supl 2):54-64.

50. Silveira EA, Martins BB, Abreu LRS, Cardoso CKS. Baixo consumo de frutas, verduras e legumes: fatores associados em idosos em capital no Centro-Oeste do Brasil. Ciência \& Saúde Coletiva. 2015;20(12):3689-3699.

51. Camelo LV, Figueiredo RC, Oliveira-Campos M, Giatti L, Barreto SM. Comportamentos saudáveis e escolaridade no Brasil: tendência temporal de 2008 a 2013. Ciência \& Saúde Coletiva. 2016;21(4):1011-1021.

52. Carvalhaes F, Chor D. Posição socioeconômica, idade e condição de saúde no Brasil. Revista Brasileira de Ciências Sociais. 2016;31(92):1-27.

53. Faleiro JC, Giatti L, Barreto SM, Camelo LV, Griep RH, Guimarães JMN, Fonseca MJM, Chor D, Chagas MCA. Posição socioeconômica no curso de vida e comportamentos de risco relacionados à saúde: ELSA-Brasil. Cadernos de Saúde Pública. 2017;33(3):e00017916.

54. Zaitune MPA, Barros MBA, Lima MG, César CLG; Carandina L, Goldbaum M, Alves MCGP. Fatores associados ao tabagismo em idosos: Inquérito de Saúde no Estado de São Paulo (ISA-SP). Cadernos de Saúde Pública. 2012;28(3):583-595.
55. Doll R, Peto R, Boreham J, Sutherland I. Mortality in relation to smoking: 50 years' observations on male british doctors. Journal of Epidemiology and Community Health. 2004;58(11):930.

56. Yang JJ, Yu D, Wen W, Shu XO, Saito E, Rahman S, Gupta PC, He J, Tsugane S, Xiang YB, et al. Tobacco smoking and mortality in Asia: A pooled meta-analysis. JAMA Network Open. 2019;2(3):1-14.

57. Pan A, Wang Y, Talaei M, Hu FB. Relation of smoking with total mortality and cardiovascular events among patients with diabetes mellitus. Circulation. 2015;132(19):1795-1804.

58. Islami F, Moreira DM, Boffetta P, Freedland SJ. A systematic review and meta-analysis of tobacco use and prostate cancer mortality and incidence in prospective cohort studies. European Urology. 2014;66(6):1054-1064.

59. Grittner U, Kuntsche S, Graham K, Bloomfield $K$. Social inequalities and gender differences in the experience of alcohol-related problems. Alcohol and Alcoholism. 2012;47(5):597-605.

60. Garcia LP, Freitas LRS. Consumo abusivo de álcool no Brasil: resultados da Pesquisa Nacional de Saúde 2013. Epidemiologia e Serviços de Saúde. 2015;24(7):227-237 